Original Research Paper

\title{
Method of Sliding Bearings Static Characteristics Calculation
}

\author{
Vladimir Nikolaevich Beschastnyh and Pavel Viktorovich Bulat \\ Saint-Petersburg National Research, University of Information Technologies, Mechanics and Optics, Saint-Petersburg, Russia
}

\author{
Article history \\ Received: 13-03-2014 \\ Revised: 27-03-2014 \\ Accepted: 01-01-2015 \\ Corresponding Author: \\ Pavel Viktorovich Bulat, \\ Saint-Petersburg National \\ Research, University of \\ Information Technologies, \\ Mechanics and Optics, Saint- \\ Petersburg, Russia \\ E-mail: pavelbulat@mail.ru
}

\begin{abstract}
The direct problem of researching the load capacity of segment gas-static bearing is being discussed. In calculation the movement of the shaft in the bearing is considered slow comparing than the rotational velocity. Vibrations and the influence of non-stationarity are neglected. The given geometry of the bearing and the pressure of lubrication supply in the gap between the bearing and the shaft are considered initial data. The result of the calculation is the value of the resultant pressure force, applied to the bearing segments. Summing the forces the value of the static load capacity can be found. For segments that may spontaneously rotate about the joint axis, described a method of calculating the rotation angle, at which the main moment of pressure forces applied to the segment is equal to zero and the segment's position is stable.
\end{abstract}

Keywords: Gas Lubrication, Gas-Static Bearing, The Reynolds Equation

\section{Introduction}

Today, the design of efficient and reliable bearings of the modern turbo-machine has become a common engineering problem, which implies the choosing of the standard design solutions according to given conditions.

The need for detailed studies arises in cases when the bearings are used under the special conditions: High or low velocities, high loads, a large number of starts, nontypical working environments (Bulat et al., 2013). In addition, the development of the sleeve bearings usually involves a detailed study of the rotor system dynamics. Study of the dynamic behavior of sliding bearings themselves is a collection of complex interdisciplinary problems and lies in the joint solution of Reynolds equation, which was first described by (Grassam and Powell, 1964) and the dynamics equation of the mechanical system, which includes a rotor and bearing with a components of external compliance and damping (Bulat and Uskov, 2012).

\section{Mathematical Model}

The main equation of hydrodynamic lubrication theory-the Reynolds equation-has no general analytical solution due to the nonlinearity. For its derivation the equations of Navier-Stokes and the equations of continuity, state and energy balance are used (Sfyris and Chasalevris, 2012).

For the turbulence model during calculation the standard laminar model is used most often (Bulat et al., 2012). The boundary of a laminar flow's existence is defined by shaft rotation speed at which the flow in the lubricating layer a stable and ordered. Stability of the flow between two coaxial cylinders was studied by Taylor (1923). Then (Schlichting, 1974) formulated stability criterion which was determined by Taylor constant:

$$
\mathrm{T}_{\mathrm{acr}}=\frac{\mathrm{u}_{\mathrm{cr}} \mathrm{H}}{\mathrm{v}} \sqrt{\psi} \leq 41,3
$$

Where:

$\mathrm{u}_{\mathrm{cr}}=$ Critical speed-the rotational velocity at the shaft's surface, at which a flow with Taylor vortices occurs

$\mathrm{H}=$ The radial gap size

$\mathrm{V}=$ The kinematic viscosity from whence

The analysis shows that for the vast majority of practical cases the velocities inside the bearings are significantly lower than $\mathrm{u}_{\mathrm{cr}}$ :

$$
\mathrm{u}_{\mathrm{cr}}=\frac{\mathrm{T}_{\mathrm{acr}} \mathrm{v}}{\mathrm{H} \sqrt{\psi}}=\frac{41,3 \mathrm{v}}{\mathrm{H} \sqrt{\psi}}
$$

It should also be noted that the flow with Taylor vortices at $\mathrm{T}_{\mathrm{a}} \geq 41,3$ is ordered and not turbulent. Developed turbulent flows can occur in non-loaded bearing areas, as well as in local elements (grooves, edges, holes).

\section{Calculation Procedure}

As a result of solving the direct problem for different variants of the lubricating layer a main resultant of pressure forces in the layer, as well as the lubrication 
consumption are determined. Constantinescu (1963) suggested the use of a stationary model of lubrication, which is still commonly used for similar calculations. But the problem can be non-stationary as well and heat exchange with the surrounding components can be taken into account.

The general methodology for performance of the checking calculations was described first by (Schlichting, 1974). For adopted bearing constructive the preliminary parameters are appointed-the pressure in the bearing cavity $\mathrm{P}_{\mathrm{a}}$, the allowed minimum gap $\mathrm{H}_{\text {min }}$, the average gap in the segment $\mathrm{H}_{0}$ (Fig. 1a) or the average gap in full-scope bearing-c.

In solving the direct problem the bearing's geometry is given constructively. The diameter of the shaft and bearing length are limited by feasibilities of the knot's placement, as well as by the limit of the rotational velocity, which is set not higher than $80 \mathrm{~m} \mathrm{~s}^{-1}$ for the radial bearing and $100 \mathrm{~m} \mathrm{~s}^{-1}$ for the periphery of the axial bearing (for oil bearing).

A three-dimensional model of the lubricating gap and surrounding components is created. The models differ from each other by the parameters of the lubricating gap. For example, for the full-scope bearing they are defined by multiple values of the eccentricity $e$ in the range of the average gap variation from $\mathrm{H}_{\min }$ to c. For radial segmental bearing $\mathrm{H}_{0}$ varies from $\mathrm{H}_{\text {min }}$ to $2 \mathrm{c}-\mathrm{H}_{\text {min }}$.

For segmental bearings the models are created with different wedge-shaped gap (often two models with different sizes of the output gap $\mathrm{H}_{\text {out }}$ are sufficient for one $\mathrm{H}_{0}$ value). Solution is made for each variation of the lubricant gap's geometry at the nominal rotation velocity of the rotor.

Then, the model, fully composed according to the steps above is forwarded to the grid generator, where a hybrid calculating grid is formed. The boundary conditions for the bearings' calculations are usually reduced to the condition of sticking at the walls and defining the pressure of the undisturbed flow in the respective sections. For this the method of creating artificial attached volumes is widely used.

The results are processed by the postprocessor, the pressure and velocities functions are visualized (Fig. 2) and the main resultant of the pressure forces, the moment relatively to the center of the joint and lubricant consumption are determined (Fig. 3).

When under no load the segments arrange concentrically to the axis of the shaft. The shaft is displaced relatively to the support's center under the influence of external force. As a result, the average gaps in the segments become different. At the place, where the gap is minimal the greatest pressure forces arise in the lubricating layer.

On the contrary, the lowest forces arise where the gap is largest.

The vector sum of the forces from all segments is the overall reaction of the bearing, which compensates the external load.

\section{Calculation of the Stable Position for Self-Aligning Segments}

Segments transmit the load to the body via the joint. Joint allows the segments to rotate about the center under the influence of hydrodynamic forces and take the position of equilibrium when the rotation moment becomes zero $\mathrm{M}_{\mathrm{z}}=0$. To find a stable position of segment for each of the current average gap $\mathrm{H}_{0}$, pressure field for two variants of the output gap $\mathrm{H}_{\text {out }}$ are calculated.

As the result of calculating all of the variants the following parameters are determined:

- Rotation moment at the center rotation, applied to the segment

- Lubrication consumption at the entrance of the segment

- The resultant of the pressure forces, applied at the segment

According to the calculations data, the diagrams of the moments and forces, depending on the output gap $\mathrm{H}_{\text {out }}$ for each current gap $\mathrm{H}_{0}$ are plotted. Then the diagram of dependence of the forces, which are applied at the segments on of the current gap size when the segment is in the equilibrium position are plotted (Fig. 4). Using these diagrams, by the method of linear interpolation the output gap $\mathrm{H}_{0 \mathrm{p}}$, at which the equilibrium position of the segment occurs and the resultant of pressure forces $\mathrm{F}_{\mathrm{K} 0}$, applied at the segment in such position are determined (Fig. 5). Load capacity of the bearing is, in general, determined by vector addition of the resultant forces, which occurs in each of the segments.

\section{Determination of Bearing Load Capacity}

Static load capacity of segmental bearing, as well as the stiffness' dependence on the eccentricity, derived from it are determined for constant or slowly changing loads (Beschastnykh and Ravikovich, 2010). When the shaft's center is displaced from the concentric position relative to the bearing by the value of eccentricity e, a certain gap $\mathrm{Hi}$ is created at each of the segments (Fig. 1b). When calculating the gaps for several values of the relative eccentricities, the resultant of pressure forces Fki is obtained for each of them (Fig. 4) and then the load capacity of the bearing is determined. Results of load capacity calculation are shown in Fig. 5.

If the segments can spontaneously rotated about the joint's axis, then theoretically there are two limiting cases: The resultant force passes through the axis of the joint or through the gap between two adjacent segments. Respective load capacities are shown in Fig. 5 in red and green. 


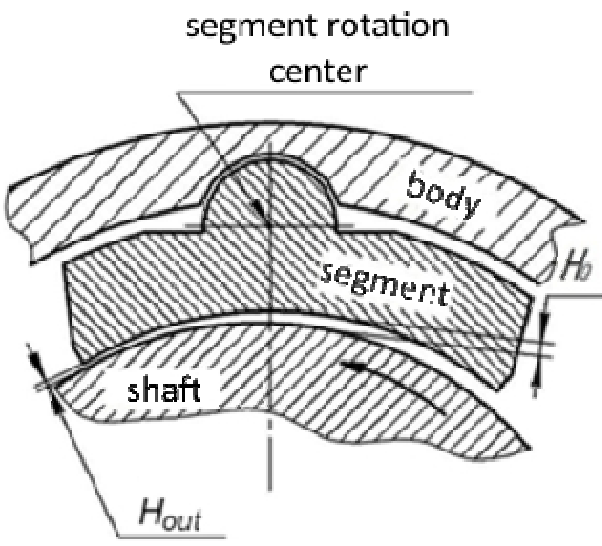

(a)

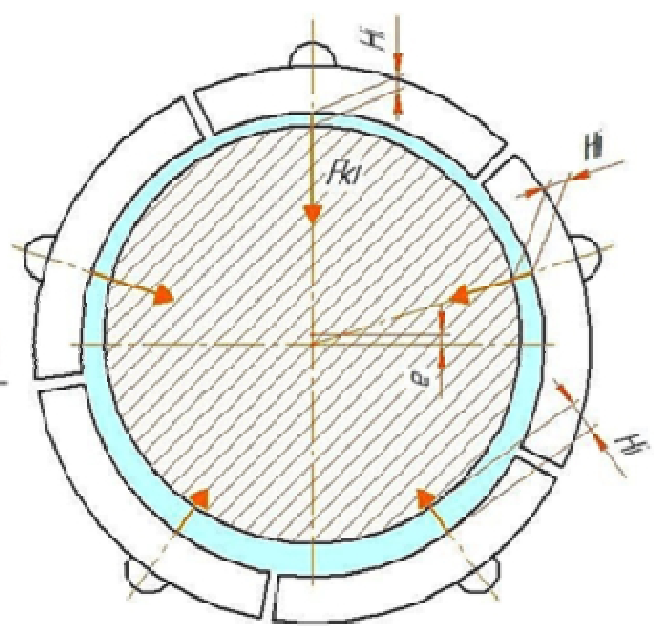

(b)

Fig. 1. Scheme of a bearing a-The segment, b-distribution of the clearances in the bearing
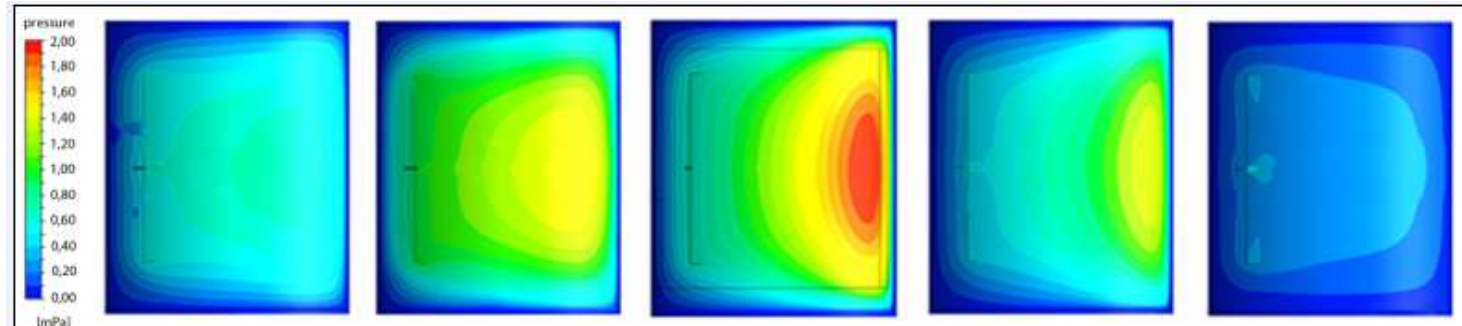

Fig. 2. Plots of pressure in the lubricating layer of the segmental bearin

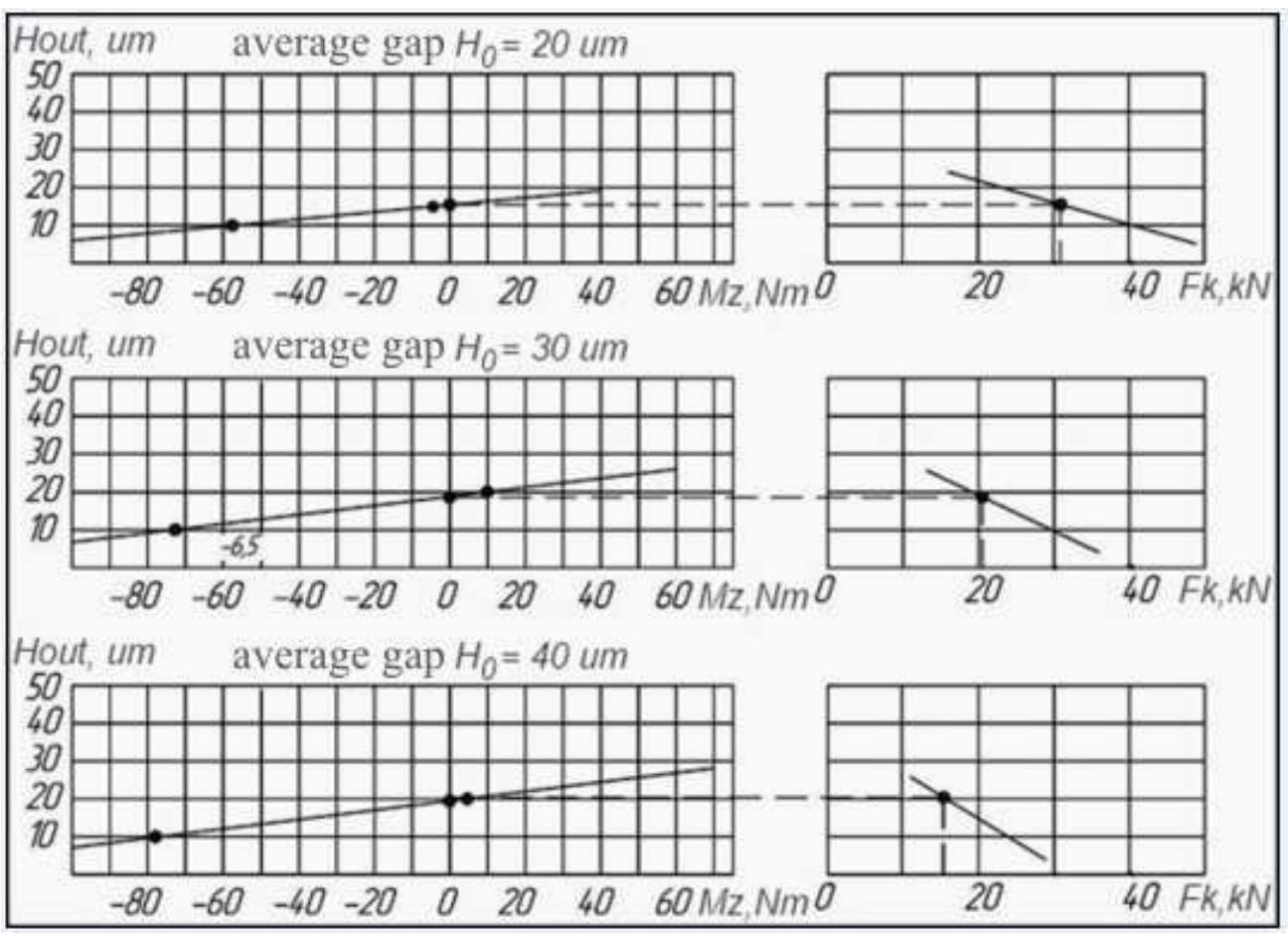

Fig. 3. The results of moments and forces calculations 


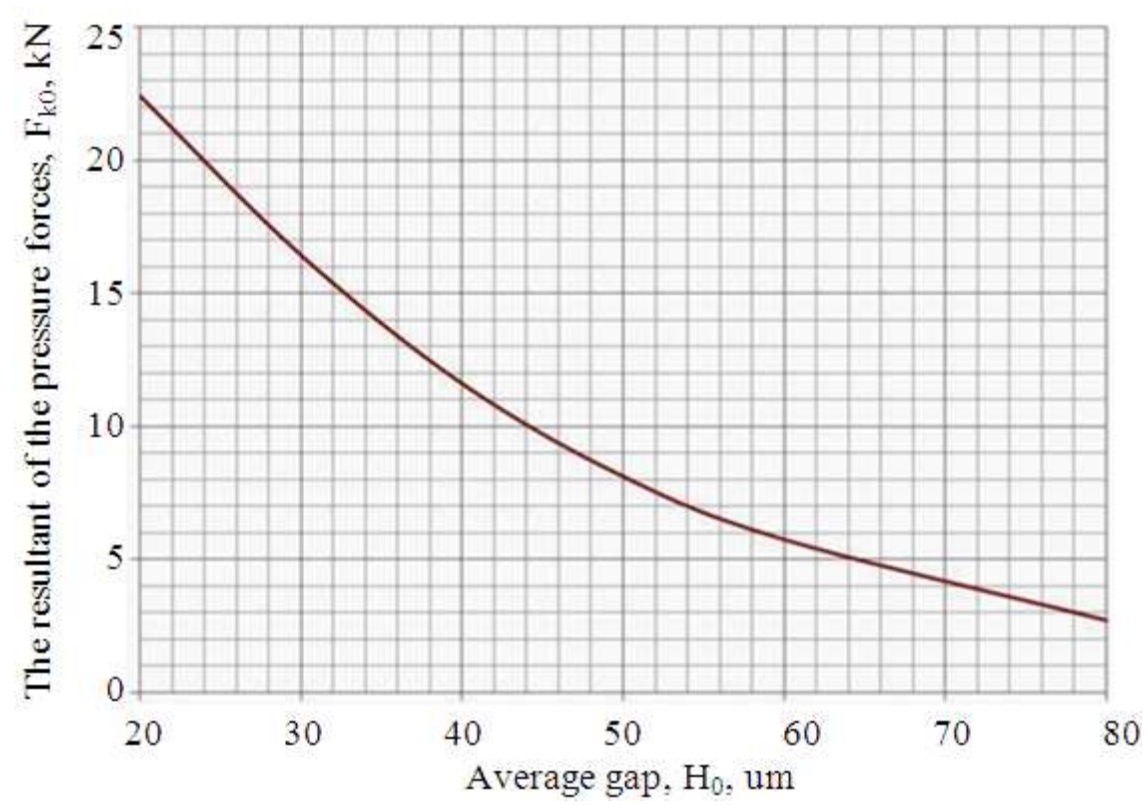

Fig. 4. Results for the segmental bearing

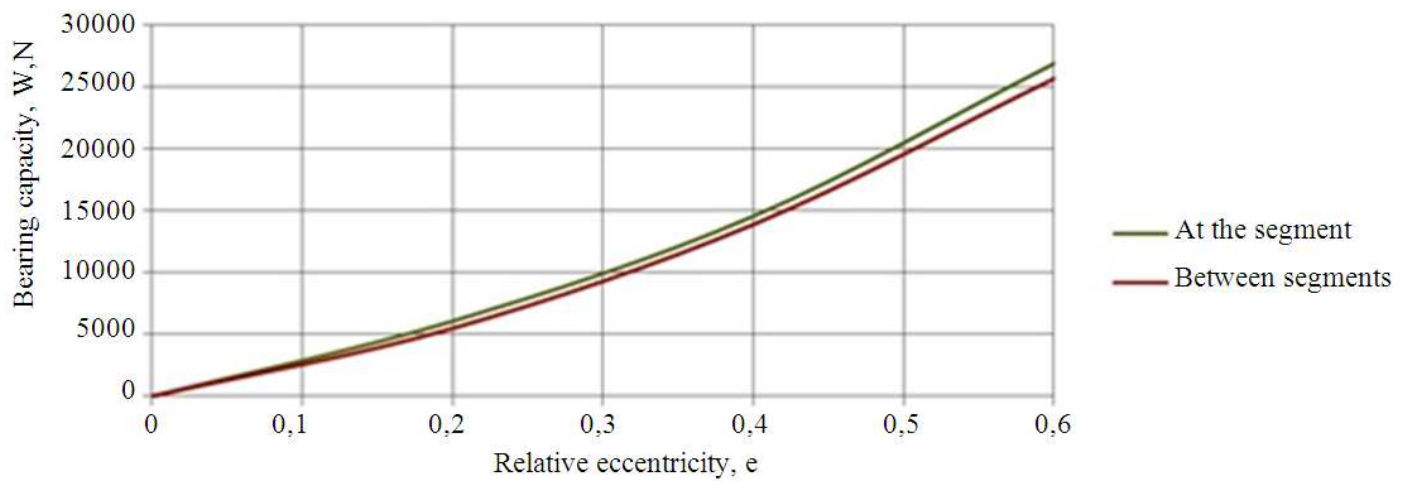

Fig. 5. Static characteristic of the segmental bearing

\section{Features of the Calculation of Dynamic Modes}

Vibrational loads, typical for turbo-machinery have the values of vibration velocities are by three orders of magnitude lesser than the rotation velocity of the shaft, which allows to omit them when calculating the static characteristics. In case of using gases as a lubricant the effect quickly increasing load has little influence on the form of characteristics, so for the analysis of rotor's dynamics the calculations of the bearing's static characteristics are performed on intermediate loads. The resulting dependences are interpolated and used later in the analysis of the rotor system dynamics.

In the case, when the lubrication is incompressible, the situation is different. Under the influence of rapidly growing loads the pressure in the lubricating layer rises much faster due to the growth of the fluid velocity, flowing out of the gap as a result of compression. In the limit, with infinitely rapid growth of the load, the incompressible fluid in the gap behaves as a solid matter. Thus, the calculations of dynamics and liquid lubrication layer must be performed together.

\section{Findings}

- A general method for calculating the static characteristics of sleeve bearings is proposed

- Recommendations on the creation of calculating models and on the assumption of the boundary conditions for the formulation of the problem regarding the study of the flow in the lubricating layer of the sleeve bearing was worked up

\section{Conclusion}

To determine the load capacity of the bearing and the stiffness of its lubricating layer the movement of the rotor is considered quasi-stationary, i.e., the characteristic gap between the bearing's segment and 
the surface of the shaft changes slowly. Changing the eccentricity of the shaft's position leads to a change in the characteristic gaps they become different for each of the segments. Specifying the sequence of eccentricities, the main moment and the resultant of pressure forces, applied to each segment are. The summation of these forces gives the value of the bearing's load capacity at the given pressure of the lubrication supply and the given eccentricity. Interpolating the obtained values, the dependence of load capacity on the average gap size is plotted. If the load is known, equating it to the capacity values, the position of the shaft in the bearing is obtained.

When self-aligning segments are used in bearing's construction, it is additionally needed to determine the angle of rotation about the axis of the joint, at which the position of the segment will be stable. To do this, a series of calculations at different angles of rotation segment are made and this allows to find the angle, at which the main moment of the forces is equal to zero.

\section{Acknowledgement}

This article was prepared as part of the " 1000 laboratories" program with the support of SaintPetersburg National Research University of Information Technologies, Mechanics and Optics.

\section{Funding Information}

This article was funded by University ITMO (SaintPetersburg National Research University of Information Technologies, Mechanics and Optics).

\section{Author's Contributions}

Pavel Viktorovich Bulat: Wrote the main text of the article, findings, coordinated the study.

Vladimir Nikolaevich Beschastnyh: Performed all the calculation work.

\section{Ethics}

Such issues are not expected.

\section{References}

Beschastnykh, V.N. and Y.A. Ravikovich, 2010. Gas bearing for heavy gas turbine rotor. Experience in the design and implementation. Vestnik Moskovskogo Aviatsionnogo Instituta, National Research University.

Bulat, P.V. and V.N. Uskov, 2012. About the survey of oscillatory motion of the gas suspended rotor for turbo-refrigerating machines and expanders. Part I. Statement of the problem. Vestnik Int. Acad. Refrigerat., 3: 3-7.

Sfyris, D. and A. Chasalevris, 2012. An exact analytical solution of the Reynolds equation for the finite journal bearing lubrication. Tribology Int., 55: 46-58. DOI: $10.1016 /$ j.triboint.2012.05.013

Bulat P.V., O.N. Zasukhin and N.V. Prodan, 2012. Features of the application of turbulence models in the calculation of flows in supersonic tracts of advanced jet engines. Dvigatel, 1: 20-23.

Schlichting, H., 1974. Boundary-Layer Theory. 1st Edn., Springer, ISBN: 10-3540662707, pp: 712.

Bulat, P.V., N.V. Prodan and O.S. Smirnova, 2013. Application of the guided gaso-and hydrostatical bearings in the turbopump aggregates of multiple combined LRE. Fundamental Res., 2: 335-339.

Constantinescu, V.N., 1963. Gas lubrication. 1st Edn., American Society of Mechanical Engineers, New York, ISBN: 10-9780608123851, pp: 645.

Grassam, N.S. and J.W. Powell, 1964. Gas Lubricated Bearing. Micro Turbine Developments Ltd. 1st Edn., London, Butterworths, ISBN: 10-0408203706, pp: 398.

Taylor, G.I., 1923. Stability of a viscous liquid contained between two rotating cylinders. Philosophical Trans. A, 223: 289-343. DOI: $10.1098 /$ rsta. 1923.0008 